\title{
Search for neutron flux generation in a plasma discharge electrolytic cell
}

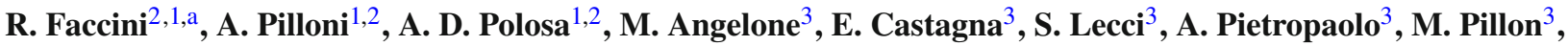 \\ M. Sansovini ${ }^{3}$, F. Sarto $^{3}$, V. Violante ${ }^{3}$, R. Bedogni ${ }^{4}$, A. Esposito ${ }^{4}$ \\ ${ }^{1}$ Dipartimento di Fisica, Sapienza Università di Roma "La Sapienza”, Piazzale Aldo Moro 2, 00185 Rome, Italy \\ 2 INFN Sezione di Roma, Piazzale Aldo Moro 2, 00185 Rome, Italy \\ ${ }^{3}$ Enea Centro Ricerche di Frascati, Via E. Fermi 45, 00044 Frascati, Italy \\ ${ }^{4}$ INFN Laboratori Nazionali di Frascati, Via E. Fermi 40, 00044 Frascati, Italy
}

Received: 4 March 2014 / Accepted: 8 May 2014 / Published online: 3 June 2014

(C) The Author(s) 2014. This article is published with open access at Springerlink.com

\begin{abstract}
Following some recent unexpected hints of neutron production in high-voltage atmospheric discharges, we present a measurement of the neutron flux in plasma discharges in electrolytic cells. We use two different types of neutron detectors, polyallyl diglycol carbonate (PADC, aka CR-39) tracers and indium disks. At $95 \%$ C.L. we provide an upper limit of 1.5 neutrons $\mathrm{cm}^{-2} \mathrm{~s}^{-1}$ for the thermal neutron flux at $\approx 5 \mathrm{~cm}$ from the center of the cell. Allowing for a higher energy neutron component, the largest allowed flux is 64 neutrons $\mathrm{cm}^{-2} \mathrm{~s}^{-1}$. This upper limit is two orders of magnitude smaller than the signal previously claimed in an electrolytic cell plasma discharge experiment. Furthermore the behavior of the CR-39 is discussed to point out possible sources of spurious signals.
\end{abstract}

\section{Introduction}

The possibility that new sources of neutrons, the so-called Low Energy Nuclear Reactions (LENR), could be devised and exploited to produce energy is a topic of paramount importance on both the applicative and theoretical grounds. Some impressive hints on neutron production in high-voltage atmospheric discharges are reported in a recent paper [1]. From the theoretical point of view, there is an open debate on whether inverse- $\beta$ nuclear transmutations could justify such observations [2-7]. Experiments are therefore needed to guide the research in the field, thus providing a sound basis for understanding the underlying physics involved in LENR phenomena.

To this end, experiments must be as accurate as possible investigating all the subtle and not yet fully understood

a e-mail: riccardo.faccini@roma1.infn.it aspects related to the subject. This work aims to clarify some still open and debated problems related to the possible emission and detection of neutrons from LERN reactions.

Here we report on a measurement performed at much smaller voltages than Ref. [1] in plasma discharges of an electrolytic cell that are claimed in the experiment [8] and in patents [9] to produce neutrons. With respect to the existing claims, we perform here a rigorous scrutiny of the detectors and the possible backgrounds to the measurement using a similar experimental setup. As neutron detectors we used the CR-39 detectors, also used in the experiment on atmospheric discharges [1], and indium activation, commonly used in neutron flux measurements. A dedicated effort was made to gain understanding of the behavior of the CR-39 detectors.

\section{The experimental setup}

The two main elements of this measurement are the electrolytic cell and the neutron detectors. For reasons that will be evident after the results have been presented, the experiment consisted of three data-taking campaigns, Run1, Run2, and Run3, that took place in February-March, June, and September 2013, respectively. The experimental setup and the changes between the campaigns will be discussed in the following.

\subsection{The electrolytic cell}

Plasma electrolysis can be considered a hybrid between the conventional electrolysis process and the atmosphericpressure plasma production. In aqueous solutions the electrochemical process can produce plasma with applied voltages of at least $\approx 130 \mathrm{~V}$. 
Fig. 1 Scheme of the experimental setup. At right a picture of the cell with plasma

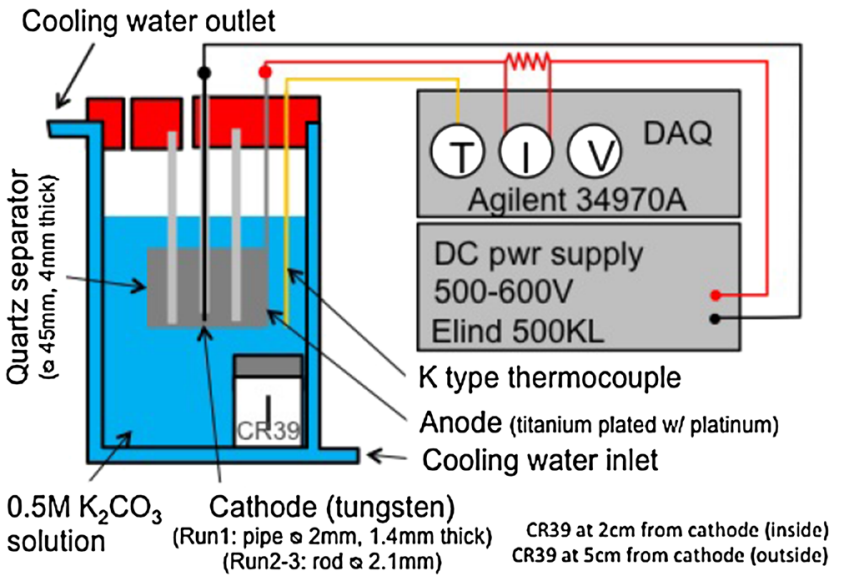

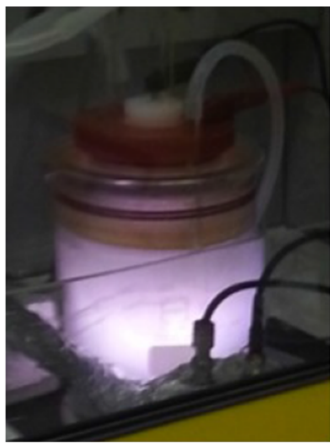

The electrochemical cell we use (see Fig. 1) consists of a water cooled pyrex vessel with the anodic and the cathodic areas separated by a quartz cylinder of $45 \mathrm{~mm}$ inner diameter, $4 \mathrm{~mm}$ thick. The anode consists of a cylindric grid of titanium plated with platinum positioned outside the quartz cylinder, while the tungsten cathode is a pipe having an outer diameter of $2 \mathrm{~mm}$ and a lateral wall thickness of $1.4 \mathrm{~mm}$ during Run 1 or a cylindrical rod with a diameter of $2.1 \mathrm{~mm}$ during Run2 and Run3. The cathode is partially covered by a quartz tube, in order to reduce its conductive active area, thus increasing the electric impedance of the electrochemical system. This makes the plasma discharge rise at the electrolyte-cathode interface. The electrolyte consists of 1.161 of $0.5 \mathrm{M} \mathrm{K}_{2} \mathrm{CO}_{3}$ aqueous solution.

The cell is covered with an araldite cap having two passages, through which the gases produced both in the cathodic zone (hydrogen and oxygen basically produced by plasma discharge) and in the anodic zone (oxygen only from the Faradic current) are separately collected. The experiment has been performed in voltage-control conditions. The power supplier is an Elind model 500KL2,4/6. The acquisition system is composed by an Agilent 34970A data logger and by a National Instruments NIDAQPad-6015. The electrolyte temperature has been measured by a $\mathrm{K}$ type thermocouple.

Plasma formation has been observed (see Fig. 1) in a voltage range from 150 to $300 \mathrm{~V}$, with current ranging from 1.5 to 3.0 A. During each run, after an initial adjustment phase, the current is verified to be stable within $\pm 25 \%$ around the average.

\subsection{The neutron detectors}

To detect the neutron flux, two different types of detectors were used: polyallyl diglycol carbonate (PADC, CR-39 in the following) track detectors and indium disks. To optimize their sensitivity they were located close to the cathode, where the plasma is produced, i.e. $5 \mathrm{~cm}$ when placed outside the cell, $2 \mathrm{~cm}$ when inside.

\subsection{The CR-39 detectors}

The CR-39 detectors produced by Intercast used in this tests are intrinsically sensitive to fast neutrons, which produce tracks on them. Thermal neutrons can also be detected by wrapping the detectors in boron: the ${ }^{10} \mathrm{~B}(\mathrm{n}, \alpha){ }^{7} \mathrm{Li}$ reaction generate $\alpha$ particles of $<1.8 \mathrm{MeV}$ that create tracks in CR-39.

The samples used during the Run1 campaign belonged to a different stock than those used during the Run2 and Run3 campaigns. Each stock is expected to have different sensitivities and background levels and will be considered separately in the following. The CR-39 detectors were coupled with a thin $(50 \mu \mathrm{m})$ layer of pure ${ }^{10} \mathrm{~B}$. Furthermore, since in the original experiment [8] the CR-39 detectors were completely covered by a thick layer of boric acid, we also used some of the Run 1 detectors with $\mathrm{a} \approx 1 \mathrm{~cm}$ thick surrounding layer of boron.

To estimate the number of neutrons that traversed the detector since its production, the CR-39 slabs are etched for $90 \mathrm{~min}$ in a $6.25 \mathrm{~N} \mathrm{KOH}$ solution at $70^{\circ} \mathrm{C}$. The number of tracks consistent with $\alpha$ particles is then estimated with an in-house automated reader consisting of an epiillumination microscope on which a 8 Mpixel CCD camera is mounted [10]. This number, divided by the detector area $\left(2.224 \mathrm{~cm}^{2}\right)$, yields the track density $(D)$.

Spurious signals or ambient backgrounds are subtracted by analyzing a set of detectors not exposed to the neutron source under study. The distributions of $D$ in the three background samples used in this study and described later are shown in Fig. 2. Indicating with $D_{b k g}$ the mean value of $D$ on such detectors we estimate the neutron flux on the irradiated detectors as $F=\left(D-D_{b k g}\right) /(c T)$ where $T$ is the duration of the exposure. The calibration 


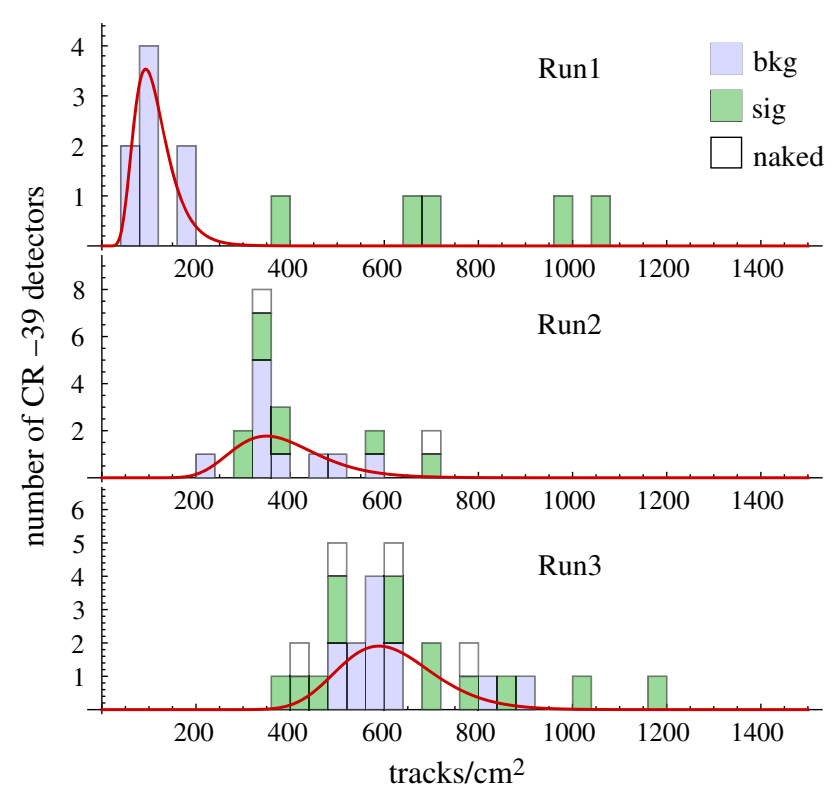

Fig. 2 Distribution of the track density $D$ in background detectors (pale blue histograms), irradiated detectors (dark green histograms) and detectors not coupled with boron (open histograms). The data from the three data-taking campaigns are separated. The lines represent the results of fits of the background distributions to a log-normal function

constant $c$ was obtained separately for the two stocks of detectors by exposing part of the detectors to a calibrated thermal neutron flux of $(1.20 \pm 0.06) \times 10^{4} \mathrm{~cm}^{-2} \mathrm{~s}^{-1}$ at the Casaccia ENEA-INMRI facility for different exposure times.

There are four systematic error contributions to such measurements: the Poisson fluctuations of the number of observed tracks, a $10 \%$ uncertainty on the number of tracks due to the counting process, the uncertainty on the calibration $c$, and the propagation of the error on $D_{b k g}$. When quoting the measured fluxes all errors will be summed in quadrature.

We also estimated the compatibility of irradiated samples with the background: we first fit the measured $D$ distributions on background with a log-normal distribution

$L(D \mid \mu, \sigma)=\frac{1}{\sqrt{2 \pi} D \sigma} \exp \left[-\frac{(\log D-\mu)^{2}}{2 \sigma^{2}}\right]$,

with $D>0$ [11] and then we estimate the probability of a measured value of $D$ not to be consistent with the background to be $\mathcal{P}(D)=\int_{0}^{D} L(x \mid \mu, \sigma) \mathrm{d} x$. To quantify the sensitivity of a negative result, when $\mathcal{P}<99 \%$ we also compute, with a Bayesian approach, the minimum neutron flux that is inconsistent with the measured track density at $95 \%$ C.L., $F_{\text {lim }}$. We would like to stress the novelty of this rigorous approach that will allow in the following to evidence features that would have not been observed otherwise.

\subsection{The indium disks}

If exposed to a neutron flux the indium produces, after the ${ }^{115} \operatorname{In}(\mathrm{n}, \gamma){ }^{116}$ In transition, mainly $1,293,1,097$, and $416 \mathrm{keV}$ $\gamma$ lines with a half life of $54 \mathrm{~min}$. The photon production cross section depends on the energy of the neutrons, ranging from $\approx 1.5$ barn for the expected spectrum from partially moderated fast neutrons ("PM" in the following) to $\approx 60$ barn for thermal neutrons.

Indium disks of a diameter of $5 \mathrm{~cm}$ and $30 \mathrm{~g}$ total mass are therefore used as neutron detectors with a two stages process: they are first exposed to a potential neutron flux and then their activity is measured by means of a high purity absolutely calibrated germanium detector of $60 \%$ efficiency. In absence of prior knowledge of the neutron spectrum, the conversion between the measured activity and the neutron flux is established by exposing the disks to a moderated AmBe neutron source of known rate $\left(\approx 2 \times 10^{6}\right.$ neutrons $\left.\mathrm{s}^{-1}\right)$ and by correcting for the decay times of the indium isotopes. It is to be noted that, due to the long half life of the involved transition, this detector works as an integrator, independently of the temporal structure of the source. In case no signal is observed, the $95 \%$ C.L. upper limit on the photon rate, and therefore on the neutron flux, is computed from the background level, the germanium detector efficiency, and the conversion factors.

As a check, an indium disk and a CR-39 detector were also simultaneously exposed to the neutrons produced by a $5.5 \times 10^{4}$ neutrons s ${ }^{-1} \mathrm{AmB}$ neutron source placed at the cathode of the electrolytic cell filled with water and not powered. The comparison with detailed simulations performed with MCNP [12] confirmed the calibration of the indium technique, while the consistency between indium disks and CR-39 detectors within 30\% showed that within this range the calibration of the CR-39 detectors holds up to PM neutrons.

We will report upper limits computed assuming a thermal neutron flux. In case of a PM neutron spectrum the limits would be a factor $\approx 40$ larger.

\section{Results of the Run1 data taking}

In our first experimental campaign the CR-39 detectors were calibrated by exposing three sets of three detectors at the ENEA-INMRI thermal neutron facility for 1, 2, and 5 min respectively. The background was estimated from a sample of eight detectors that were not irradiated and not wrapped with boron and that were analyzed at the same time as the calibration samples. From a linear fit to the dependence of the measured tracks density after background subtraction on the known neutron flux, the calibration constant is estimated to be $c=(6.9 \pm 0.3) \times 10^{-3}$ tracks/neutrons. 
Table 1 Characteristics of the runs and results. CR-39 detectors marked with (inside) were placed inside the cells; the ones marked with $(\mathrm{Al})$ and $(\mathrm{Cd})$ were wrapped in aluminum and cadmium. Upper limits are at $95 \%$ C.L. The indium upper limits are computed assuming a thermal neutron flux. For a PM neutron spectrum the limits are a factor $\approx 40$ larger

\begin{tabular}{|c|c|c|c|c|}
\hline Run & Duration (') & Voltage range $(\mathrm{V})$ & n-flux (In) $\left(\mathrm{n} \mathrm{cm}^{-2} \mathrm{~s}^{-1}\right)$ & n-flux (CR-39) $\left(\mathrm{n} \mathrm{cm}^{-2} \mathrm{~s}^{-1}\right)$ \\
\hline \multirow[t]{2}{*}{$1 \mathrm{~A}$} & \multirow[t]{2}{*}{8} & \multirow[t]{2}{*}{$150-200$} & & $280 \pm 32$ (inside) \\
\hline & & & & $259 \pm 30$ (inside) \\
\hline 1B & 5 & 250 & $<1.5$ & $275 \pm 35$ \\
\hline $1 \mathrm{C}$ & 12 & $150-200$ & $<0.7$ & $109 \pm 14$ (inside) \\
\hline 1D & 4 & $150-300$ & & $166 \pm 32$ (inside) \\
\hline \multirow[t]{3}{*}{2} & \multirow[t]{3}{*}{13} & \multirow[t]{3}{*}{$220-300$} & \multirow[t]{3}{*}{$<0.6$} & $<49,<14$ \\
\hline & & & & $<23,<18(\mathrm{Al})$ \\
\hline & & & & $<16,<21(\mathrm{Cd})$ \\
\hline \multirow[t]{3}{*}{$3 \mathrm{~A}$} & \multirow[t]{3}{*}{20} & \multirow[t]{3}{*}{150} & \multirow[t]{3}{*}{$<0.4$} & $<26,<27$ \\
\hline & & & & $89 \pm 15(\mathrm{Al})$ \\
\hline & & & & $<19,<5(\mathrm{Cd})$ \\
\hline \multirow[t]{2}{*}{ 3B } & \multirow[t]{2}{*}{21} & \multirow[t]{2}{*}{$200-300$} & \multirow[t]{2}{*}{$<0.4$} & $\begin{array}{l}<11,39 \pm 12 \\
<39,<8(\mathrm{Cd})\end{array}$ \\
\hline & & & & $56 \pm 13(\mathrm{Al})$ \\
\hline
\end{tabular}

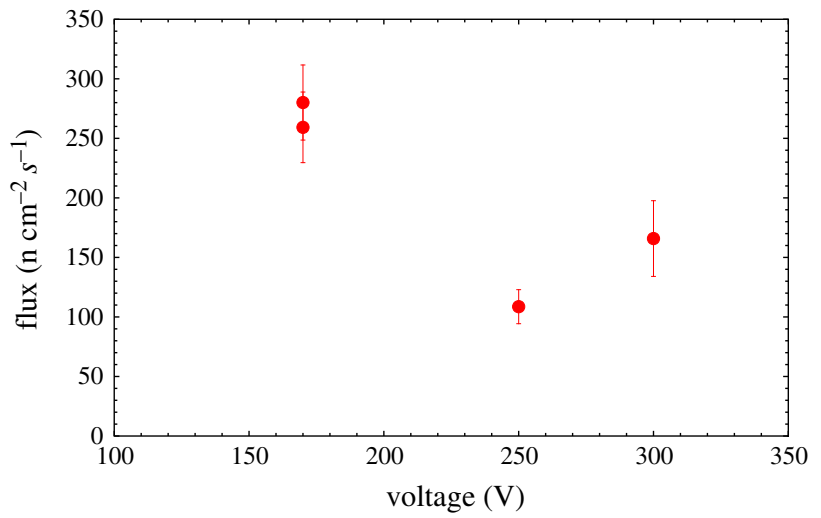

Fig. 3 Dependence of the neutron flux, as estimated from the track density excess in Run1 data, on the average voltage applied to the electrolytic cell

At the same time, CR-39 detectors covered with a thick boron coating were exposed to the calibration flux, but no significant signal was observed: the boron indeed absorbs the neutrons and stops the $\alpha$ particles. Indeed the detectors used in Ref. [8] have a calibration constant which is $\approx 70$ times smaller, thus confirming the scarce sensitivity of that measurement.

Several weeks afterwards we performed four different runs where we placed neutron detectors both close to the electrolytic cell and inside it. The runs differed by voltage applied to the cell, by duration, and by type and number of CR-39 detectors located inside and outside the cell. During two of these runs we also used the indium disks. The results are summarized in Table 1, where a letter is appended to Run1 to distinguish among the configurations. In analyzing the results we used the same background estimated for cal- ibration, which means that the exposed detectors were analyzed 40 days after the corresponding background ones.

As shown in Table 1 and Fig. 2, the detectors presented a small but significant deviation from the background. Such a deviation did not show any dependence on whether the detector was placed inside or outside the cell, nor on the voltage (see Fig. 3). Even attributing this excess to neutrons produced in the cell, the measured fluxes range between 110 and 280 neutrons $\mathrm{cm}^{-2} \mathrm{~s}^{-1}$, incompatibly with the $\approx 72,000$ neutrons $\mathrm{cm}^{-2} \mathrm{~s}^{-1}$ reported in Ref. [8].

On the contrary, neither indium disks nor CR-39 detectors with thick boron coating placed both close to the cathode and outside the cell ever measured any signal, establishing that the thermal (fast) neutron flux is smaller than 1.5 (64) neutrons $\mathrm{cm}^{-2} \mathrm{~s}^{-1}$ at $95 \%$ C.L.

\section{CR-39 background studies}

The inconsistency between the results obtained with the CR39 and the indium techniques led us to further investigate the background subtraction in the analysis of the CR-39 detectors. In the Run1 analysis the background was treated differently from the signal because it had not been wrapped with boron and it was analyzed 40 days before the exposed detectors.

To this aim, in the Run2 and Run3 campaigns the background detectors were covered with the thin boron film and analyzed simultaneously with the exposed detectors. Furthermore, we studied the time evolution of the background by measuring it at a distance of 60 days. Figure 2 shows a significant increase of background with time (from Run2 to Run3) that we estimated to be $\mathrm{d} D / \mathrm{d} t=3.8$ tracks $\mathrm{cm}^{-2} \mathrm{day}^{-1}$. 
Furthermore, detectors without boron, marked as "naked" in Fig. 2, behave like the background ones. This suggests that the background is not due to thermal neutrons, but rather either they have a cosmic origin [13], or they are due to radon contamination. From Ref. [14] we estimated that in the city of Naples, where the experiment [8] was performed, the average radon contamination would fake on average on CR-39 detectors $\approx 13$ tracks $\mathrm{cm}^{-2}$ day $^{-1}$, i.e. a contribution even larger than the one we measured. Assuming the same calibration as thermal neutrons, we concluded that such a background has a rate that can easily cause significant systematic errors if not properly accounted for. We thus considered the results of the CR-39 detectors during the Run1 data taking flawed.

\section{Results of the Run2 and Run3 data taking}

We have performed the last two data-taking campaigns with the same setup but separated by two months, paying attention to a consistent treatment of exposed and background detectors. To study the nature of a possible excess, we had wrapped some of the detectors in aluminum (to screen electromagnetic radiation) and cadmium (to screen thermal neutrons). The calibration constant used is $(8.3 \pm 0.8) \times 10^{-3}$ tracks/neutrons; see above.

The results of both the CR-39 and the indium detectors, see Table 1 and Fig. 2, show that the exposed detectors yield track densities consistent with the background. The two outliers are those wrapped in aluminum during Run3, as though the wrapping could cause some spurious tracks. Considering conservatively the detector with the largest deviation from the background, we set a limit on the neutron flux $\Phi<105$ neutrons $\mathrm{cm}^{-2}$ day $^{-1}$ from the CR-39 detectors and $\Phi<0.6$ (26) neutrons $\mathrm{cm}^{-2}$ day $^{-1}$ from the indium disks assuming a thermal (PM) neutron spectrum.

\section{Conclusions}

Following the observation of neutron production in highvoltage discharges [1], we have investigated the same phenomenon at lower voltages, in plasma discharges inside an electrolytic cell, by rigorously verifying the claims of an experiment [8] and of patents [9]. With respect to the existing measurements we have optimized and studied in detail the neutron detectors (CR-39 tracers and indium disks) and properly have taken into account the ambient background and its fluctuations.

In particular, we verified that the boron layer on the CR39 detectors needs to be $\approx 50 \mu \mathrm{m}$ thick to avoid loss of sensitivity to thermal neutrons, and that CR-39 detectors, regardless of the presence of boron, integrate $\mathrm{d} D / \mathrm{d} t=3.8$ tracks $\mathrm{cm}^{-2}$ day $^{-1}$, likely due to cosmic radiation and radon contamination. It is therefore critical to pay attention to the treatment of the background, since a delay in analyzing the irradiated detectors with respect to the background ones could lead to false positives. Furthermore, we confirmed that non-irradiated CR-39 detectors show long tails in the track density distribution [11], and we noted a small spurious signal in detectors wrapped in aluminum. Therefore, especially when very low neutron fluxes are expected, the use of CR-39 detectors should be accompanied by careful consideration of disturbing effects.

After taking all these effects in account, from the absence of signals in the indium disks, which are the most sensitive device, we conclude that the produced neutron flux is smaller than 1.5 (64) neutrons $\mathrm{cm}^{-2} \mathrm{~s}^{-1}$ at $95 \%$ C.L. assuming a thermal (PM) neutron spectrum. From the measurements with CR-39 detectors in Run2 and Run3, when the background was treated properly, we can exclude fluxes larger than 105 neutrons $\mathrm{cm}^{-2} \mathrm{~s}^{-1}$ at $95 \%$ C.L. Such limits are at least two orders of magnitude smaller than the measured fluxes in Ref. [8] where the detector sensitivity is smaller and the background fluctuations are ignored.

Acknowledgments We thank L. Maiani and G. Ruocco for stimulating discussions and S. Loreti for the CR-39 calibration (INMRI-ENEA, Rome, Italy).

Open Access This article is distributed under the terms of the Creative Commons Attribution License which permits any use, distribution, and reproduction in any medium, provided the original author(s) and the source are credited.

Funded by $\mathrm{SCOAP}^{3}$ / License Version CC BY 4.0.

\section{References}

1. A.V. Agafonov et al., Phys. Rev. Lett. 111, 115003 (2013)

2. A. Widom, L. Larsen, Eur. Phys. J. C 46, 107 (2006)

3. Y.N. Srivastava, A. Widom, L. Larsen. http://arxiv.org/abs/0810. 0159

4. A. Widom, Y.N. Srivastava, L. Larsen. http://arxiv.org/abs/0804. 2647

5. A. Widom, Y.N. Srivastava, L. Larsen. http://arxiv.org/abs/0709. 1222

6. A. Widom, L. Larsen. http://arxiv.org/abs/nucl-th/0608059

7. S. Ciuchi et al., Eur. Phys. J. C 72, 2193 (2012)

8. D. Cirillo, R. Germano, V. Tontodonato, A. Widom, Y.N. Srivastava, E. Del Giudice, G. Vitiello, Key Eng. Mater. 495, 104 (2012)

9. Patent US 8419919 B1, System and methods for generating particles. See also patent WO 1999049471 A1, Reactor for producing energy and neutrons by electrolytic reaction in light- or heavy-water solution

10. R. Bedogni et al., Radiat. Meas. 43, S491 (2008)

11. R. Bedogni et al., Radiat. Meas. 43, 1108 (2008)

12. R. Arthur Forster et al., Nucl. Inst. Meth. B 213, 82 (2004)

13. M.S. Gordon et al., IEEE Trans. Nucl. Sci. 51, 3427 (2004)

14. Matiullah, J. Radioanal. Nucl. Chem. 298 (2013). http://www.a2c. it/Radon-Rn-222/banca-dati-misurazioni-radon.html 